Mitteilungen aus dem chemischen Institut der Universität Berlin.

\title{
Synthese von Polypeptiden XXXII.
}

\section{Derivate der Asparaginsäure;}

von Emil Fischer und Albert Fiedler.

(Eingelaufen am 11. Juli 1910).

Obschon die Asparaginsäure in den natürlichen Proteinen bei weitem nicht in so großen Mengen wie die Glutaminsäure vorkommt, so ist sie doch als älteste und einfachste Aminodicarbonsäure so wichtig, daß die Kenntnis ihrer Polypeptide für das Studium der Proteine wünschenswert erscheint. Wir haben deshalb die Reaktionen, die bei der Glutaminsänre zum Aufbau des Tetrapeptides Glycylglutamyldiglycin dienten, ${ }^{1}$ ) auf die Asparaginsäure übertragen und so in der Tat ein Tetrapeptid aus $3 \mathrm{Mol}$. Glykokoll und $1 \mathrm{Mol}$. Asparaginsäure erhalten, dem wir nach der Synthese folgenden Namen und Formel geben wollen -

\section{Glycyl-asparagyl-diglycin, $\mathrm{NH}_{2} \mathrm{CH}_{2} \mathrm{CO}$. NHCHCO.NHCH${ }_{2} \mathrm{COOH}$ $\mathrm{CH}_{2} \mathrm{CO} . \mathrm{NHCH}_{2} \mathrm{COOH}$.}

Leider findet beim Übergang von der aktiven Chloracetyl-l-asparaginsäure zu dem Chloracetylasparagyldiglycinäthylester eine Racemisierung statt, welche wahrscheinlich durch die Behandlung der Säure mit Phosphorpentachlorid veranlaBt wird. Jedenfalls waren der von uns isolierte Ester und alle daraus weiter hergestellten Produkte einschließlich des Tetrapeptids optisch inaktiv.

1) E. Fischer, W. Kropp und A. Stahlschmidt, diese Annalen 365, 181 (1909). 
Nach bekannten Methoden haben wir ferner noch dargestellt l-Leucylglycyl-l-asparaginsäure und l-Leucyl1-asparaginsäure. Eine Lencylasparaginsäure ist schon früher beschrieben worden. ${ }^{1}$ ) Da sie aber aus l-Asparaginsäureester und optisch inaktivem $\alpha$-Bromisocapronylchlorid hergestellt wurde, so war sie kein optisch einheitlicher Körper, sondern ein Gemisch der Stereoisomeren, die hier entstehen müssen.

Dieser Schluß wird durch den später angeführten optischen Vergleich des neuen Präparates mit dem alten Produkt bestätigt.

\section{Chloracetyl-l-asparaginsäure, $\mathrm{ClCH}_{2} \mathrm{CO}$. NHCH. $(\mathrm{COOH}) \mathrm{CH}_{2}(\mathrm{COOH})$.}

Zur Darstellung dient entweder Asparaginsäure oder Asparagin. Das Verfahren ist im wesentlichen dasselbe wie bei der Bereitung der Chloracetylglutaminsäure. Wir wollen es hier ausführlich nur für das Asparagin beschreiben, das wegen der Billigkeit für größere Operationen zu bevorzugen ist.

$100 \mathrm{~g}$ trocknes Asparagin oder $113 \mathrm{~g}$ krystallwasserhaltiges Material (1 Mol.) werden mit $758 \mathrm{ccm} 3 \mathrm{n}$-Natronlauge ( 3 Mol.) in einer Schale etwa $1 / 2$ Stunde stark gekocht, bis kein Ammoniak mehr entweicht, und mithin die Umwandlung in Asparaginsäure vollzogen ist. Die hierbei ziemlich stark konzentrierte Lösung versetzt man nach dem Abkühlen mit $151 \mathrm{ccm} 5$ n-Salzsäure, um $1 / 3$ der ursprünglich angewandten Natronlauge zo neutralisieren. Ihr Volum beträgt dann $400-500 \mathrm{ccm}$. Man kühlt nun sorgfältig durch Kältemischung und fügt im Laufe von einer Stunde unter starkem Schütteln in etwa sechs Portionen abwechselnd $100 \mathrm{~g}$ Chloracetylchlorid (1,2 Mol.) und $760 \mathrm{ccm}$ eiskalte 2n-Natronlange (2 Mol.) hinzu. Der Geruch des Säurechlorids verschwindet jedesmal ziemlich rasch. Nach Eintragen der letzten Portion

1) E. Fi scher und E. Königs, Ber. d. d. chem. Ges. 37, 4593 (1904). 
läßt man die Lösung noch etwa eine Stunde in der Kälte stehen. In der Flüssigkeit ist zum Schluß die Chloracetylasparaginsäure als Natriumsalz vorhanden. Um sie in Freiheit zu setzen, bzw. alles Natrium an Chlor zu binden, sind $425 \mathrm{ccm} 5 \mathrm{n}$-Salzsäure notwendig. Man setzt aber von dieser Menge nur so viel zu, bis die Flüssigkeit auf Lackmus sauer reagiert, und verdampft dann unter $12-15 \mathrm{~mm}$ Druck möglichst rasch auf etwa $300 \mathrm{ccm}$. Jetzt wird der Rest der Salzsäure zugefügt, abgekühlt und das ausgeschiedene Kochsalz scharf abgesaugt. Die Mutterlauge wird unter gleichem Druck aus einem Bad von etwa $40^{\circ}$ ganz zur Trockne verdampft, während das abfiltrierte Kochsalz im Vakuumexsiccator ebenfalls getrocknet wird. Zur Isolierung der Chloracetylasparaginsäure ist die Extraktion mit heißem Essigäther am meisten zu empfehlen. Sie wird sowohl auf den trocknen Rückstand wie auf das zuerst auskrystallisierte Kochsalz, das immer etwas Chloracetylverbindung einschliebt, angewandt. Es ist nötig, vor der Extraktion die Masse zu pulverisieren. Sie wird dann wiederholt mit ungefähr $500 \mathrm{ccm}$ Essigäther $10 \mathrm{Mi}-$ nuten am Rückflußkühler gekocht. Zweckmäßig ist es, das zuerst abgeschiedene Kochsalz getrennt zu behandeln, ferner die essigätherische Lösung jedesmal erst nach dem Abkühlen zu filtrieren und endlich die Auszüge sofort abzndestillieren, nm das Destillat für neue Extraktionen zu verwenden. Nach dem Verdampfen des Essigäthers bleibt ein hellgelbes Öl, das sich in der Kälte in einen dicken Brei von Krystallen verwandelt. Die Krystallisation wird durch Impfen sehr beschleunigt. Nach etwa 12 stündigem Stehen wird die Krystallmasse scharf abgesaugt und mit wenig eiskaltem Essigäther nachgewaschen.

Zur Reinigung löst man in etwa 9 Gew.-Tln. Essigäther, entfärbt die schwachgelbe Flüssigkeit mit wenig Tierkohle, und verdampft das Filtrat auf die Hälfte. In der Kälte fällt die Chloracetylasparaginsäure, besonders 
nach dem Impfen, als farbloses, krystallinisches Pulver. Die eingeengte Mutterlauge gibt auf Zusatz von Petroläther eine zweite, viel geringere Krystallisation. Die Gesamtausbeute an umkrystallisierter Substanz betrug ungefähr $118 \mathrm{~g}$ oder 74 Proz. der Theorie.

Für die Analyse war nochmals aus Essigäther umkrystallisiert und bei $78^{\circ}$ unter $12 \mathrm{~mm}$ Druck über Phosphorpentoxyd getrocknet.

$0,2116 \mathrm{~g}$ gaben $0,2672 \mathrm{CO}_{2}$ und $0,0744 \mathrm{H}_{2} \mathrm{O}$.

$0,1910 \mathrm{~g}$ gaben (über 33 prozentiger Natronlauge) 10,9 ccm Stickgas bei $17^{\circ}$ und $763 \mathrm{~mm}$ Druck.

0,1732 g gaben 0,1173 $\mathrm{AgCl}$.

$\begin{array}{ccr} & \text { Ber. für } \mathrm{C}_{6} \mathrm{H}_{8} \mathrm{O}_{5} \mathrm{NCl}(209,53) & \text { Gef. } \\ \mathrm{C} & 34,36 & 34,45 \\ \mathrm{H} & 3,85 & 3,93 \\ \mathrm{~N} & 6,69 & 6,66 \\ \mathrm{Cl} & 16,92 & 16,75\end{array}$

Die Substanz schmilzt im Capillarrohr unter Gasentwickelung gegen $142-143^{\circ}$ (korr.). Sie Jöst sich sehr leicht in Wasser und Alkohol. In Äther und namentlich Petroläther ist sie fast gar nicht löslich. Die mit Ammoniak neutralisierte wäßrige Lösung gibt mit Silbernitrat einen farblosen, amorphen Niederschlag, der sich bei dem Erhitzen der Flüssigkeit in erheblicher Menge löst.

$0,8009 \mathrm{~g}$ Substanz. Gesamtgewicht der wäBrigen Lösung 7,9543 g. $d^{19}=1,0367$. Drehung im $2 \mathrm{dm} \cdot$ Rohr bei $19^{\circ}$ und Natriumlicht $0,87^{\circ}$ nach rechts. Mithin

$$
[\alpha]_{\mathrm{D}}^{19}=4,17^{\circ}( \pm 0,2) \text {. }
$$

$0,5116 \mathrm{~g}$ Substanz. Gesamtgewicht der Lösung 5,0875 g. $\mathrm{d}^{19}=1,0384$. Drehung im $1 \mathrm{dm}-$ Rohr bei $19^{\circ}$ und Natriumlicht $0,44^{\circ}( \pm 0,02)$ nach rechts. Mithin

$$
[x]_{\mathrm{D}}^{19}=+4,21( \pm 0,2) \text {. }
$$

Zum Beweis, daß die Substanz keine wesentliche Menge von Racemkörper enthielt, haben wir sie durch Salzsäure hydrolysiert und das Drehungsvermögen der hierdurch entstehenden Lösung bestimmt. 
$1 \mathrm{~g}$ Chloracetylasparaginsäure (entsprechend $0,6363 \mathrm{~g}$ Asparaginsäure) wurde mit $5 \mathrm{ccm} 20$ prozentiger Salzsäure 5 Stunden auf $100^{\circ} \mathrm{im}$ geschlossenen Rohr erhitzt. Die Drehung der Lösung war dann im 1-dm-Rohr 2,9 $9^{\circ}$ nach rechts, $d^{19}=1,101$, während eine Lösung von $0,6363 \mathrm{~g}$ reiner l-Asparaginsäure in $5 \mathrm{ccm} 20$ prozentiger Salzsäure mit $\mathrm{d}^{18}=1,118$ den Wert $3,09^{\circ}$ gab.

\section{Glycyl-l-asparaginsäure, $\mathrm{NH}_{2} \dot{\mathrm{CH}}_{2} \mathrm{CO}$. $\mathrm{NHCH}(\mathrm{COOH}) \cdot \mathrm{CH}_{2}(\mathrm{COOH})$.}

Wird eine Lösung von $10 \mathrm{~g}$ Chloracetylverbindung in $50 \mathrm{ccm}$ wäßrigem Ammoniak von 25 Proz. 3 Tage bei Zimmertemperatur aufbewahrt, so ist die Abspaltung des Halogens beendet. Zur Isolierung des Dipeptids müssen Halogen und Ammoniak völlig entfernt werden. $\mathrm{Zu}$ dem $\mathrm{Zweck}$ fügt man eine warme Lösung von $30 \mathrm{~g}$ krystallisiertem Bariumhydroxyd zu und verdampft unter $12-15 \mathrm{~mm}$ Druck auf etwa die Hälfte, bis alles Ammoniak verschwunden ist. Zur Entfernung des Chlors wird jetzt auf bekannte Weise mit Schwefelsäure und Silbersulfat gefällt und die völlig von Chlor, Silber, Barium und Schwefelsäure befreite Flüssigkeit unter geringem Druck eingedampft. Wird der zurückbleibende Sirup in einer Platinschale einigemal unter Umrühren mit absolutem Alkohol verdampft, so verwandelt er sich in eine feste amorphe Masse. Die Ausbeute an Rohprodukt beträgt ungefähr 80 Proz. der Theorie.

Um daraus Krystalle zu gewinnen, löst man in Wasser und fugt Alkohol bis zur Trübung zu. Bei mehrtägigem Stehen beginnt in der Regel die Krystallisation. In dem Maße, wie sie fortschreitet, fügt man in gelinder Wärme $\left(35-40^{\circ}\right)$ mehr Alkohol, immer bis zur Trübung, zu. Durch Impfen wird die Operation beschleunigt, immerhin kann sie bei größeren Mengen etwa 2 Wochen dauern. Zur völligen Reinigung genügt es, die abgesaugten Krystalle nochmals in der 5 fachen Menge Wasser zu lösen, mit wenig Tierkohle zu erhitzen und das warme 
Filtrat wiederum mit Alkohol bis zur Trübung zu versetzen. Beim Impfen geht jetzt die Krystallisation so rasch vonstatten, daß sie in 24 Stunden beendet sein kann. Die Ausbeute an reinem, zweimal krystallisiertem Dipeptid betrug 65 Proz. d. Th. Das krystallinische Pulver zeigte unter dem Mikroskop keine charakteristische Form.

Wenn das Dipeptid aus verdünnter Lösung krystallisiert ist, so enthält es in der Regel 1 Mol. Wasser, das bei $105^{\circ}$ und $12 \mathrm{~mm}$ Druck über Phosphorpentoxyd rasch weggeht.

$0,1936 \mathrm{~g}$ über Schwefelsäure im Vakuum getrocknet, verloren bei $105^{\circ}$ unter $12 \mathrm{~mm}$ Druck $0,0170 \mathrm{~g}$.

$0,2002 \mathrm{~g}$ verloren $0,0174 \mathrm{~g}$.

Ber. für $\mathrm{C}_{6} \mathrm{H}_{10} \mathrm{~N}_{2} \mathrm{O}_{5} \cdot \mathrm{H}_{2} \mathrm{O}(208,1)$
$\mathrm{H}_{2} \mathrm{O}$
8,65
$8,78 \quad 8,69$

Gef.

Das trockne Dipeptid gab folgende Zahlen:

$0,1828 \mathrm{~g}$ gaben $0,2531 \mathrm{CO}_{2}$ und $0,0883 \mathrm{H}_{2} \mathrm{O}$.

$0,2020 \mathrm{~g} "$ nach Kjeldahl $21 \mathrm{cem}{ }^{\mathrm{n}} /{ }_{10}-\mathrm{NH}_{3}$.

$\begin{array}{ccr} & \text { Ber. für } \mathrm{C}_{6} \mathrm{H}_{10} \mathrm{~N}_{2} \mathrm{O}_{5}(190,1) & \text { Gef. } \\ \mathrm{C} & 37,87 & 37,76 \\ \mathrm{H} & 5,30 & 5,40 \\ \mathrm{~N} & 14,74 & 14,56\end{array}$

Ans konzentrierten Lösungen haben wir einigemal Präparate erhalten, die viel weniger Wasser (2 Proz.) enthielten, und die wohl ein Gemisch von wasserhaltiger und wasserfreier Substanz waren.

Für die optische Bestimmung diente ebenfalls ein bei $105^{\circ}$ getrocknetes und vorher mehrmals umkrystallisiertes Präparat.

$0,2730 \mathrm{~g}$ Substanz, gelöst in Wasser. Gesamtgewicht 2,7903 g. $\mathrm{d}^{20}=1,044$. Drehung im $1 \mathrm{dm}-\mathrm{Rohr}$ bei $20^{\circ}$ und Natriumlicht $1,13^{\circ}( \pm 0,01)$ nach rechts. Mithin

$$
[\alpha]_{\mathrm{D}}^{20}=+11,06^{\circ}( \pm 0,1) \text {. }
$$

$0,6254 \mathrm{~g}$ nochmals umkrystallisierte Substanz. Gesamtgewicht der Lösung 6,7880. $\mathrm{d}^{20}=1,0404$. Drehung in $1 \mathrm{dm}$-Rohr bei $20^{\circ}$ und Natriumlicht $1,06^{\circ}( \pm 0,01)$ nach rechts. Mithin

$$
[\alpha]_{\mathbf{D}^{0}}^{2^{0}}=+11,1^{0}( \pm 0,1) \text {. }
$$


Das getrocknete Dipeptid schmilzt bei raschem Erhitzen im Capillarrohr gegen $203^{\circ}$ (korr. 207 ${ }^{\circ}$ ) unter starker Gasentwickelung nud Gelbfärbung.

Es ist in Wasser leicht löslich und reagiert stark sauer. In kaltem Alkohol ist es fast unlöslich. Die mit Ammoniak neutralisierte wäßrige Lösung gibt mit Silbernitrat einen dicken, amorphen, farblosen Niederschlag, der sich beim Erwärmen der Flüssigkeit in erheblicher Menge löst. Das Dipeptid löst Kupferoxyd beim Kochen mit tiefblauer Farbe.

\section{d-ce-Bromisocapronyl-glycyl-l-asparaginsäure,} $\left(\mathrm{CH}_{3}\right)_{2} \mathrm{CHCH}_{2} \mathrm{CHBrCO}_{\mathrm{NHCH}} \mathrm{CO} . \mathrm{NHCH}(\mathrm{COOH}) \mathrm{CH}_{2} \mathrm{COOH}$.

In eine Lösung von $10 \mathrm{~g}$ Glycyl-l-asparaginsäure (1 Mol.) und 105,5 ccm n-Natronlauge (2 Mol.), die durch eine Kältemischung gekühlt ist, trägt man unter starkem Schütteln in sechs Portionen and abwechselnd $13,5 \mathrm{~g}$ (1,2 Mol.) d- $\alpha$-Bromisocapronylchlorid (aus d-Leucin) und $105,5 \mathrm{ccm}$ gekühlte n-Natronlange im Laufe von etwa 2 Stunden ein. Nach weiterem einstündigem Stehen wird mit $29,5 \mathrm{ccm} 5 \mathrm{n}$-Salzsäure $(2,8 \mathrm{Mol}$.) angesäuert. Es ist dabei zweckmäßig, zunächst nur einen Teil der Säure bis zur dauernden Trübung zuzusetzen und jetzt einige Kryställchen einzutragen, um sofort die Krystallisation einzuleiten. Fügt man dann allmählich den übrigen Teil der Salzsäure zu, so erreicht man, daß der Bromkörper gar nicht als Öl, sondern völlig krystallisiert ausfällt. Die ersten Krystalle muß man sich allerdings anf mühsamerem Wege, durch Extraktion des Öls mit Äther, Trocknen und Verdampfen der Lösung und längeres Stehen des Rückstandes im Vakuumexsiccator bereiten.

Wenn die wäßrige Flüssigkeit nach Zusatz der gesamten Salzsäure 12 Stunden im Eisschrank gestanden hat, werden die Krystalle scharf abgesangt, im Vakuumexsiccator getrocknet und mit Petroläther gewaschen, um kleine Mengen von Bromisocapronsäure zu entfernen. Die Ausbeute beträgt etwa 12,5 g. Eine kleine Menge 
(etwa $1 \mathrm{~g}$ ) desselben Produktes läßt sich aus der sauren Mutterlauge gewinnen, indem man sie unter sehr geringem Druck einengt und das abgeschiedene Öl ansäthert, dann den Äther trocknet, verdampft und den Rückstand durch längeres Stehen und Zusatz von Petroläther zur Krystallisation bringt.

Zur Reinigung wird die Substanz in etwa 5 Tln. heißen Wassers gelöst, mit wenig Tierkohle entfärbt und das Filtrat auf $0^{\circ}$ abgekiuhlt. Sie fällt dann fast vollständig in kurzen Prismen aus. An diesem reinen Produkt wurden im ganzen $13 \mathrm{~g}$ aus $10 \mathrm{~g}$ Dipeptid gewonnen.

Die Krystalle enthalten Wasser. Für die lufttrockne Substanz fanden wir seine Menge entsprechend ungefähr $1 / 2$ Mol. Im Vakuumexsiccator über Schwefelsäure geht es bei zwölfstündigem Stehen nicht weg, wohl aber bei mehrstündigem Trocknen unter $15 \mathrm{~mm}$ Druck über Phosphorpentoxyd bei $78^{\circ}$.

$0,2592 \mathrm{~g}$ (über Schwefelsäure getrocknet) verloren $0,0061 \mathrm{~g}$.

$0,2299 \mathrm{~g}$ verloren $0,0051 \mathrm{~g}$.

Ber. für $\mathrm{C}_{12} \mathrm{H}_{19} \mathrm{O}_{6} \mathrm{~N}_{2} \mathrm{Br}+1 / 2 \mathrm{H}_{2} \mathrm{O}(376,1)$

Gef.

$\mathrm{H}_{2} \mathrm{O} \quad 2,39$

$2,35 \quad 2,22$

Die trockne Substanz gab folgende Zahlen:

$0,1607 \mathrm{~g}$ gaben $0,2305 \quad \mathrm{CO}_{2}$ und $0,0758 \mathrm{H}_{2} \mathrm{O}$.

$0,1594 \mathrm{~g}, \quad 10,3 \mathrm{ccm}$ Stickgas bei $18^{\circ}$ und $766 \mathrm{~mm}$ Druck über 33 prozentiger Natronlauge.

$0,1577 \mathrm{~g}$ gaben $0,0815 \mathrm{AgBr}$.

Ber. für $\mathrm{C}_{12} \mathrm{H}_{19} \mathrm{O}_{6} \mathrm{~N}_{2} \mathrm{Br}(367,09) \quad$ Gef.

$\mathrm{C} \quad 39,23 \quad 39,12$

$\begin{array}{lrr}\mathrm{H} & 5,22 & 5,28\end{array}$

$\begin{array}{lll}\mathrm{Br} & 21,77 & 21,98\end{array}$

$\begin{array}{lll}\mathrm{N} & \mathbf{7 , 6 4} & \mathbf{7 , 5 5}\end{array}$

Der trockne Körper ist hygroskopisch. Die im Exsiccator getrocknete Substanz schmilzt im Capillarrohr bei $118-119^{\circ}\left(119-120^{\circ}\right.$ korr.). Er löst sich in ungefähr 4 Tln. heißem Wasser, auch leicht in Alkohol, dagegen schwer in Äther und Benzol.

Für die optische Bestimmung diente eine alkoholische Lösung. 
$0,6472 \mathrm{~g}$ getrocknete Substanż. Gesamtgewicht der Lösung $5,6656 \mathrm{~g}$. $\mathrm{d}^{21}=0,8374$. Drehung im $1 \mathrm{dem}-$ Rohr bei $21^{\circ}$ und Natriumlicht $5,88^{\circ}( \pm 0,02)$ nach rechts. Mithin

$$
[\boldsymbol{\alpha}]_{\mathrm{D}}^{21}=+61,5^{0}( \pm 0,2) \text {. }
$$

$0,4048 \mathrm{~g}$ Substanz. Gesamtgewicht der Lösung 4,0501 g. $\mathrm{d}^{21}=0,8328$. Drehung im $1 \mathrm{dem}-R o h r$ bei $21^{\circ}$ und Natriumlicht $5,09^{\circ}( \pm 0,02)$ nach rechts. Mithin

$$
[\alpha]_{\mathrm{D}}^{21}=+61,2^{0}( \pm 0,2) \text {. }
$$

l-Leucylglycyl-l-asparaginsäure, $\mathrm{C}_{4} \mathrm{H}_{8} \cdot \mathrm{CH}\left(\mathrm{NH}_{2}\right) \mathrm{CO} \cdot \mathrm{NHCH}{ }_{2} \mathrm{CO} \cdot \mathrm{NHCH}(\mathrm{COOH}) \cdot \mathrm{CH}_{2} \mathrm{COOH}$.

Die Darstellung aus dem Bromkörper geschah genau so, wie bei der Glycyl-l-asparaginsäure beschrieben ist. Bei dem schließlichen Verdampfen der wäßrigen Lösung bleibt ein Sirup zurück. Er wird mit wenig Wasser in eine Platinschale gespült, anf dem Wasserbad wieder eingedampft und durch Verreiben mit absolutem Alkohol in eine amorphe Masse verwandelt. Nach dem Abfiltrieren und Trocknen betrug seine Menge 75 Proz. d. Th. Um das Produkt krystallinisch zu erhalten, löst man $2 \mathrm{~g}$ in einer Platinschale in wenig warmem Wasser, fügt $60 \mathrm{ccm}$ Alkohol zu und verdampft unter Umrühren auf dem Wasserbad. Diese Operation muß fünf- bis sechsmal wiederholt werden, bis in der Hitze die Abscheidung von Krystallen beginnt, und bei dem Erkalten die Lösung keine Gallerte mehr abscheidet. Läßt man dann die auf etwa $30 \mathrm{ccm}$ eingeengte Flüssigkeit langsam erkalten, so fällt das Tripeptid in mikroskopisch kleinen, farblosen Nadeln aus. Sie werden abgesaugt, in wenig Wasser gelöst und die Flüssigkeit bei $50^{\circ}$ mit Aceton bis zur dauernden Trübung versetzt. Beim langsamen Abkühlen fällt das reine Tripeptid in feinen Nadeln aus. Seine Menge betrug ungefähr $2 / 3$ des amorphen Rohprodukts.

Für die Analyse und optische Untersuchung wurde bei $105^{\circ}$ und $12-15 \mathrm{~mm}$ Druck über Phosphorpentoxyd getrocknet.

$0,1315 \mathrm{~g}$ gaben $0,2283 \mathrm{CO}_{2}$ und $0,0836 \mathrm{H}_{2} \mathrm{O}$.

$0,2061 \mathrm{~g} 川 20,1 \mathrm{ccm}{ }^{\mathrm{n}} / 10^{-}-\mathrm{NH}_{\mathrm{g}}$. 


$\begin{array}{lcr}\text { Ber. für } & \mathrm{C}_{12} \mathrm{H}_{21} \mathrm{O}_{6} \mathrm{~N}_{3}(303,2) & \text { Gef. } \\ \mathrm{C} & 47,49 & 47,35 \\ \mathrm{H} & 6,98 & 7,11 \\ \mathrm{~N} & 13,86 & 13,65\end{array}$

$0,0740 \mathrm{~g}$ Substanz. Gesamtgewicht der wäBrigen Lösung 1,4390 g. $d^{20}=1,017$. Drehung im $1 \mathrm{dem}$-Rohr bei $20^{\circ}$ und Natriumlicht $2,89^{\circ}( \pm 0,02)$ nach rechts. Mithin

$$
[\alpha]_{\mathrm{D}}^{20}=+55,25^{\circ}[ \pm 0,2] \text {. }
$$

$0,1709 \mathrm{~g}$ Substanz. Gesamtgewicht der Lösung 3,2411 g. $\mathrm{d}^{20}=1,018$. Drehung im $1 \mathrm{dem}-\mathrm{Rohr}$ bei $20^{\circ}$ und Natriumlicht $2,95^{\circ}( \pm 0,02)$ nach rechts. Mithin

$$
[\alpha]_{\mathrm{D}}^{2^{0}}=+54,96^{\circ}[ \pm 0,2] \text {. }
$$

Bei raschem Erhitzen im Capillarrohr schmilzt das Tripeptid gegen $233^{\circ}$ (corr. $239^{\circ}$ ) unter Zersetzung nach vorhergehender Bräunung.

Es löst sich in Wasser sehr leicht und reagiert sauer. In absolutem Alkohol und Aceton ist es sehr wenig löslich.

\section{Chloracetyl-asparagyl-diglycyläthylester, $\mathrm{ClCH}_{2} \mathrm{CO}$. $\mathrm{NHCHCO} . \mathrm{NHCH}_{2} \mathrm{COOC}_{2} \mathrm{H}_{5}$ $\mathrm{CH}_{2} \mathrm{CO} . \mathrm{NHCH}_{2} \mathrm{COOC}_{2} \mathrm{H}_{5}$.}

$10 \mathrm{~g}$ sorgfältig getrocknete und sehr fein gepulverte Chloracetylasparaginsäure werden mit $50 \mathrm{ccm}$ frisch destilliertem Acetylchlorid in einem mit Glasstopfen versehenen Schüttelzylinder übergossen, auf etwa $5^{0}$ abgekühlt und dann $22 \mathrm{~g}(2,2 \mathrm{Mol}$.) rasch gepulvertes Phosphorpentachlorid in drei Portionen unter Schütteln und im Laufe von 15 Minuten eingetragen. Hierbei findet fast vollständige Lösung der Chloracetylasparaginsäure statt. Man schüttelt jetzt noch 15 Minuten bei Zimmertemperatur, kühlt dann auf $0^{\circ}$ ab und filtrier rom unverbrauchten Phosphorpentachlorid in dieselbe Flasche, in der nachher die Filtration vorgenommen wird. Wird jetzt das Acetylchlorid unter möglichst geringem Druck verdampft, so scheidet sich in der Regel das Säurechlorid als dicke, aber nicht deutlich 
krystallinische Masse ab. Man verdünnt mit $150 \mathrm{ccm}$ scharf getrocknetem Petroläther, um die Abscheidung des Chlorids zu vervollständigen und filtriert die Masse sofort mit einem Pukallschen Tonfilter unter Benutzung des für diesen $Z$ weck früher empfohlenen Apparats. ${ }^{1}$ )

Nachdem noch zweimal mit je $50 \mathrm{ccm}$ trocknem Petroläther nachgewaschen ist, wird das Produkt im Vakuumexsiccator über Phosphorpentoxyd getrocknet und bildet dann ein farbloses, lockeres Pulver. Die Ausbeute betrug gewöhnlich $6,2 \mathrm{~g}$ oder 52 Proz. d. Th.

Wenn beim Abdestillieren des Acetylchlorids das Süurechlorid sich nicht ausscheidet, so wird die auf etwa $20 \mathrm{ccm}$ eingeengte Flüssigkeit allmählich und unter Reiben mit etwa $200 \mathrm{ccm}$ Petroläther verdünnt, wobei die Abscheidung eintritt.

Das so gewonnene Chlorid ist keineswegs rein. Eine Chlorbestimmung ergab nur 24,1 Proz., während 28,8 Proz. berechnet sind. Trotzdem haben wir es direkt für die Kuppelung mit Glykokollester benutzt. Za dem Zweck wurden $6 \mathrm{~g}$ Rohprodukt mit $50 \mathrm{ccm}$ ganz trocknem Äther übergossen. Dabei geht das Chlorid in Lösung. Man gießt die ätherische Lösung von dem Rückstand ab, der zum Teil aus Chloracetylasparaginsäure besteht und spült mit $50 \mathrm{ccm}$ Äther nach. Diese Lösung wird sofort zu einer durch Eis gekühlten Mischung von $15 \mathrm{~g}$ frisch destilliertem Glykokollester und $50 \mathrm{ccm}$ trocknem Äther unter Schütteln zugetropft. Der hierbei sofort entstehende Niederschlag ist ein Gemisch von salzsaurem Glykokollester und Chloracetylasparagindiglycinester. Er wird abgesangt, mit Äther gewaschen, nach dem Trocknen im Vakuumexsiccator zur Entfernung des salzsauren Glykokollesters mit wenig eiskaltem Wasser verrieben, abgesangt und mit kaltem Wasser gewaschen.

Die Ausbeute an rohem Chloracetylasparagyldiglycinäthylester betrug 6,9 g. Zur Reinigung wird das Pro-

1) Ber, d. d. chem. Ges. 38, 616 (1905). 
dukt in der 20 fachen Gewichtsmenge heißem Essigäther gelöst und mit wenig Tierkohle entfärbt. Aus dem Filtrat scheidet sich in der Kälte der reine Chloracetylasparagyldiglycinester in farblosen, sehr dünnen biegsamen Nadeln aus. Die Ausbente betrug nur $4,3 \mathrm{~g}$ oder 24 Proz. d. Th., berechnet auf die angewandte Chloracetylasparaginsäure. Aus der Essigäther-Mutterlauge gewinnt man durch Einengen noch eine kleine Menge $(0,2 \mathrm{~g})$. Beim völligen Verdampfen hinterläßBt sie ein dickes Öl.

Zur Analyse wurde nochmals aus heißem Essigäther umgelöst und unter $15 \mathrm{~mm}$ Druck bei $78^{\circ}$ getrocknet.

$0,1568 \mathrm{~g}$ gaben $0,2532 \mathrm{CO}_{2}$ und $0,0806 \mathrm{H}_{2} \mathrm{O}$.

$0,2909 \mathrm{~g} \quad, \quad 0,1120 \mathrm{AgCl}$.

$0,2009 \mathrm{~g} \# 19,8 \mathrm{ccm}$ Stickgas bei $16^{\circ}$ und $745 \mathrm{~mm}$ Druck (über 33 prozentiger Natronlauge).

$\begin{array}{lcr}\text { Ber. für } & \mathrm{C}_{14} \mathrm{H}_{22} \mathrm{O}_{7} \mathrm{~N}_{3} \mathrm{Cl}(379,66) & \text { Gef. } \\ \text { C } & 44,25 & 44,04 \\ \text { II } & 5,84 & 5,75 \\ \mathrm{Cl} & 9,34 & 9,52 \\ \text { N } & 11,07 & 11,28\end{array}$

Die Substanz schmilzt im Capillarrohr bei 173 bis $174^{\circ}$ (korr. 176-177 ) nach vorhergehender Sinterung. In warmem Wasser ist sie ziemlich leicht löslich. Von heißem Essigäther sind ungefähr $17 \mathrm{ccm}$ für $1 \mathrm{~g}$ nötig, in Äther ist sie sehr schwer löslich

Die 5 prozentige wässrige Lösung zeigte im 1-dcm-Rohr keine wahrnehmbare Drehung. Ebenso inaktiv blieb die Lösung von $1 \mathrm{~g}$ des Esters in $5 \mathrm{ccm} 20$ prozentiger Salzsäure, nachdem sie 5 Stunden auf $100^{\circ}$ erhitzt war. Da hierbei Asparaginsäure entsteht, die in Salzsäure stark dreht, so beweist der Versuch, daß der Ester vollständig racemisiert ist. Wir glauben, daß diese Racemisierung vorzugsweise bei der Verwandlung der Chloracetylasparaginsäure ins Chlorid vor sich geht. 


\section{Chloracetyl-asparagyl-diglycin, $\mathrm{ClCH}_{2} \mathrm{CO}$. $\mathrm{NHCHCO}$. $\mathrm{NHCH}_{2} \mathrm{COOH}$ $\mathrm{CH}_{2} \mathrm{CO} . \mathrm{NHCH}_{2} \mathrm{COOH}$.}

Werden $5 \mathrm{~g}$ fein gepulverter Ester mit $30 \mathrm{ccm}$ n-Natronlauge $(2,2 \mathrm{Mol}$.) bei gewöhnlicher Temperatur auf der Maschine geschüttelt, so geht er im Laufe von etwa 2 Stunden völlig in Lösung. Man läßt noch $1 / 2$ Stunde stehen, versetzt dann mit $6 \mathrm{ccm} 5 \mathrm{n}$-Salzsäure und filtriert die etwas trübe Flüssigkeit schnell. In dem Filtrat beginnt besonders beim Reiben bald die Krystallisation des Chloracetylasparagyldiglycins in mikroskopischen farblosen Nadeln oder sehr dünnen Prismen. Nach zweistündigem Stehen bei $0^{0}$ wird filtriert. Die Ausbeute betrug $3,6 \mathrm{~g}$ und die eingeengte Mutterlange gab noch weiter $0,4 \mathrm{~g}$. Die gesamte Ausbente entsprach also 95 Proz. d. Th.

Zur Reinigung wird aus etwa 7 Tln. heißem Wasser umkrystallisiert. Für die Analyse diente ein zweimal umgelöstes Präparat. Die lufttrocknen Krystalle enthalten $1 \mathrm{Mol}$. Wasser, das schon im Vakuumexsiccator über Schwefelsäure weggeht.

$$
\begin{array}{cccc}
0,4695 \mathrm{~g} \text { verloren } & 0,0255 \mathrm{H}_{2} \mathrm{O} . & \\
0,3318 \mathrm{~g} & 0,0177 \mathrm{H}_{2} \mathrm{O} . & \\
\text { Ber. für } & \mathrm{C}_{10} \mathrm{H}_{14} \mathrm{O}_{7} \mathrm{~N}_{9} \mathrm{Cl} . \mathrm{H}_{2} \mathrm{O}(341,6) & \text { Gef. } \\
\mathrm{H}_{2} \mathrm{O} & 5,28 & 5,44 & 5,33
\end{array}
$$

Zur Analyse wurde noch bei $78^{\circ}$ über Phosphorpentoxyd bei $12-15 \mathrm{~mm}$ Druck getrocknet.

$$
\begin{aligned}
& 0,1586 \mathrm{~g} \text { gaben } 0,2154 \mathrm{CO}_{2} \text { und 0,0614 } \mathrm{H}_{2} \mathrm{O} \text {. } \\
& 0,2447 \mathrm{~g} \quad 0,1077 \mathrm{AgCl} \text {. }
\end{aligned}
$$

$0,1510 \mathrm{~g} \quad, \quad 16,8 \mathrm{ccm}$ Stickgas bei $15^{\circ}$ und $745 \mathrm{~mm}$ Druck

(über 33 prozentiger Natronlange).

$\begin{array}{lrr}\text { Ber. für } \mathrm{C}_{10} \mathrm{H}_{14} \mathrm{O}_{7} \mathrm{~N}_{3} \mathrm{Cl}(323,06) & \text { Gef. } \\ \mathrm{C} & 37,08 & 37,04 \\ \mathrm{H} & 4,36 & 4,33 \\ \mathrm{Cl} & 10,96 & 10,89 \\ \mathrm{~N} & 12,99 & 12,80\end{array}$

Die im Vakunmexsiccator getrocknete Verbindung schmilzt nicht ganz konstant im Capillarrohr gegen 


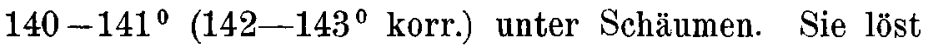
sich in 6-7 Tln. heißem Wasser, aber schwer in kaltem Wasser. In Alkohol ziemlich leicht löslich, in Essigäther und Äther recht schwer löslich. Sie reagiert stark sauer. Die mit Ammoniak neutralisierte wäßrige Lösung gibt mit Silbernitrat einen dicken, farblosen, nicht deutlich krystallisierten Niederschlag.

\section{Glycyl-asparagyl-diglycin, $\mathrm{NH}_{2} \mathrm{CH}_{2} \mathrm{CO} . \mathrm{NHCHCO.NHCH} \mathrm{COOH}_{2}$ $\mathrm{CH}_{2} \mathrm{CO} . \mathrm{NHCH}_{2} \mathrm{COOH}$.}

Eine Lösung von $5 \mathrm{~g}$ Chloracetylasparagyldiglycin in $25 \mathrm{ccm}$ Ammoniak von 25 Proz. wird 5 Tage bei $25^{\circ}$ aufbewahrt. Lann werden Ammoniak, Halogen usw. in der für Glycylasparaginsäure beschriebenen Weise entfernt. Es ist dabei ratsam, das Verdampfen immer bei möglichst geringem Druck vorzunehmen. Schließlich wird die mit Tierkohle entfärbte wäßrige Lösung, nachdem sie auf etwa $50 \mathrm{ccm}$ eingeengt ist, im Vakuumexsiccator zum dünnen Sirup verdunstet und mit wenig Aceton verrieben. Nach längerer Zeit pflegt dann das Tetrapeptid zu krystallisieren. Impfen befördert den Vorgang. Nach Beginn der Krystallisation läßt man noch 3 Tage im Eisschrank stehen, saugt dann die Krystalle ab und wäscht mit sehr wenig eiskaltem Wasser. Bei gut gelungener Operation war die Ausbeute 60 Proz. der Theorie.

Zur Reinigung löst man in etwa der 10 fachen Menge Wasser, filtriert und verdunstet das Filtrat wiederum im Exsiccator. Das Tetrapeptid scheidet sich dann in mikroskopisch feinen, farbiosen Nädelchen ab, die meist zu größeren Klumpen verwachsen sind. Die im Vakuumexsiccator über Schwefelsäure getrocknete Substanz enthielt noch etwas Wasser und wurde deshalb unter $15 \mathrm{~mm}$ Druck bei $125^{\circ}$ über Phosphorpentoxyd getrocknet.

$0,1774 \mathrm{~g}$ gaben $0,2551 \mathrm{CO}_{2}$ und $0,0860 \mathrm{H}_{2} \mathrm{O}$.

$0,1815 \mathrm{~g} \quad, 28,7 \mathrm{ccm}$ Stickgas bei $19^{\circ}$ und $766 \mathrm{~mm}$ Druck (über 33 prozentiger Natronlauge). 


$\begin{array}{lcr} & \text { Ber. für } \mathrm{C}_{10} \mathrm{H}_{16} \mathrm{O}_{7} \mathrm{~N}_{4}(304,17) & \text { Gef. } \\ \mathrm{C} & 39,45 & 39,23 \\ \mathrm{H} & 5,30 & 5,43 \\ \mathrm{~N} & 18,42 & 18,39\end{array}$

Die im Exsiccator getrocknete Substanz schmilzt bei raschem Erhitzen im Capillarrohr nach vorhergehender Sinterung und Gelbfärbung gegen $197-199^{\circ}$ (korr. 201-203\%. Das Tetrapeptid ist in Wasser, besonders in der Wärme, recht leicht löslich, dagegen in Alkohol und Aceton äußerst schwer löslich. Es reagiert und schmeckt sauer. Die mit Ammoniak neutralisierte wäßrige Lösung gibt mit Silbernitrat einen dicken, farblosen, amorphen Niederschlag, der sich beim Erhitzen der Flüssigkeit ziemlich leicht löst.

Die alkalische Lösung des Tetrapeptids gibt mit nicht zuviel Kupfersulfat eine schön rotviolette Färbung, die bei mehr Kupfersalz in Blänlichviolett übergeht.

Die wäßrige Lösung des Tetrapeptids färbt sich beim Kochen mit Kupferoxyd tiefblau. Dabei scheint aber, zumal wenn man länger kocht, Hydrolyse einzutreten.

\section{d-a-Bromisocapronyl-l-asparaginsäure,} $\left(\mathrm{CH}_{3}\right)_{2} \mathrm{CHCH}_{2} \mathrm{CHBrCO}$. NHCH$(\mathrm{COOH}) \cdot \mathrm{CH}_{2} \mathrm{COOH}$.

Die Kuppelung wurde mit $10 \mathrm{~g}$ Asparaginsäure und 19,2 $\mathrm{g}$ (1,2 Mol.) d- $\alpha$-Bromisocapronylchlorid in derselben Weise ausgeführt, wie es zuvor bei der Glycylasparaginsäure beschrieben ist. Das Kuppelungsprodukt fällt beim 24 stündigen Stehen der angesäuerten Flüssigkeit zum größten Teil $(14 \mathrm{~g})$ in feinen Nadeln aus, die fast immer zu kugeligen Aggregaten verwachsen sind. Aus der Mutterlauge wurde durch Finengen unter vermindertem

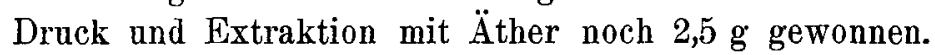
Die Gesamtausbeute betrug also 16,5 g, oder, auf Asparaginsäure berechnet, 70 Proz. der Theorie.

Zur Reinigung wurde zunächst in warmem Äther gelöst und mit Petroläther gefällt, und die so erhaltene Masse nochmals aus 5 Tln. heißem Wasser umkrystallisiert, 
wobei es nötig war, zuletzt anf $0^{\circ}$ abzukühlen und die Mutterlauge noch besonders zn verarbeiten. Die Ausbeute an reiner Säure betrug $14 \mathrm{~g}$.

Zur Analyse war im Vakuumexsiccator über Schwefelsäure getrocknet.

$$
\begin{aligned}
& 0,1826 \mathrm{~g} \text { gaben } 0,2590 \mathrm{CO}_{2} \text { und } 0,0839 \mathrm{H}_{2} \mathrm{O} \text {. } \\
& 0,2023 \mathrm{~g}, \quad 0,1232 \mathrm{AgBr} \text {. } \\
& 0,3068 \mathrm{~g} \quad, \quad 9,5 \mathrm{ccm}{ }^{\mathrm{n}} / 10^{-} \mathrm{NH}_{3} \text {. } \\
& \begin{array}{lcr} 
& \text { Ber. für } \mathrm{C}_{10} \mathrm{H}_{16} \mathrm{O}_{5} \mathrm{NBr}(310,06) & \text { Gef. } \\
\mathrm{C} & 38,70 & \mathbf{3 8 , 6 8} \\
\mathrm{H} & 5,20 & 5,14 \\
\mathrm{Br} & 25,78 & \mathbf{2 5 , 9 2} \\
\mathrm{N} & 4,51 & \mathbf{4 , 3 4}
\end{array}
\end{aligned}
$$

Die Substanz schmilzt im Capillarrohr gegen $148^{\circ}$ (korr. $150^{\circ}$ ). Sie ist nicht allein in heißem Wasser, sondern auch in Alkohol und Aceton recht leicht löslich. Auch von Äther und Essigäther wird sie in der Wärme reichlich aufgenommen. In Wasser von $20^{\circ}$ ist sie schon so schwer löslich, daß eine in der Wärme bereitete 9 prozentige Lösung beim längeren Stehen Krystalle abscheidet.

Für die optische Bestimmung diente deshalb eine ziemlich verdünnte wäßrige Lösung.

$0,4481 \mathrm{~g}$ Substanz. Gesantgewicht der Lösung 10,9536 g. $\mathrm{d}^{22}=1,013$. Drehung im $1 \mathrm{dcm}$-Rohr bei $22^{\circ}$ und Natriumlicht $0,34^{\circ}( \pm 0,02)$ nach rechts. Mithin

$$
[\alpha]_{\mathrm{D}}^{2}=+8,21^{\circ}( \pm 0,2) \text {. }
$$

$0,3186 \mathrm{~g}$ Substanz. Gesamtgewicht $6,6241 \mathrm{~g} . \quad \mathrm{d}^{22}=1,014$. Drehung im $1 \mathrm{dcm}-R o h r$ bei $22^{\circ}$ und Natriumlicht $0,39^{\circ}$ $( \pm 0,02)$ nach rechts. Mithin

$$
[\alpha]_{\mathrm{D}}^{2}=+8,00^{\circ}( \pm 0,2) \text {. }
$$

Im ganzen gleicht die Substanz sehr dem unter gleichem Namen von E. Fischer und E. Königs beschriebenen Körper ${ }^{1}$ ), der aber nach der Darstellung aus inaktiver $\boldsymbol{c}$-Bromisocapronsäure ein Gemisch von zwei Stereoisomeren sein mußte. Eine mit dem alten Präparate 
von Dr. Königs ausgeführte optische Bestimmung bestätigt diesen Schluß; denn es drehte unter den oben angegebenen Bedingungen nach links, und zwar

$$
[\alpha]_{\mathrm{D}}=-9,7^{0} \text {. }
$$

\section{l-Leucyl-l-asparaginsäure,}

$\left(\mathrm{CH}_{3}\right)_{2}$. $\mathrm{CH} \cdot \mathrm{CH}_{2} \cdot \mathrm{CH}\left(\mathrm{NH}_{2}\right) \mathrm{CO} \cdot \mathrm{NH}(\mathrm{COOH}) \mathrm{CH}_{2} \mathrm{COOH}$.

Für Darstellung und Isolierung gilt wieder die vorher für Glycylasparaginsäure gegebene Vorschrift. Nur muß die ammoniakalische Lösung des Bromkörpers bei Zimmertemperatur etwa 6 Tage stehen, bis alles Halogen abgespalten ist. Beim Verdampfen der letzten wäbrigen Lösung bleibt das Dipeptid als Sirup, der aber beim Verreiben mit absolutem Alkohol bald zu einer amorphen, farblosen Masse erstarrt. Ausbeute etwa 75 Proz. der Theorie.

Zur Reinigung wird das Produkt in wenig Wasser gelöst, mit Tierkohle in der Wärme entfärbt und das Filtrat im Exsiccator verdunstet, wobei das Dipeptid in feinen, farblosen Nadeln ausfällt. Die Ansbeute betrug ungefähr 55 Proz. der Theorie.

Die Krystalle enthalten Wasser. Für die lufttrockne Substanz fanden wir seine Menge entsprechend $2 \mathrm{Mol}$. Im Vakuumexsiccator über Phosphorpentoxyd und unter $12 \mathrm{~mm}$ Druck verliert es bei 24 stündigem Stehen das Wasser.

$$
\begin{array}{llll}
0,3474 \mathrm{~g} & \text { verloren } & 0,0443 & \mathrm{H}_{2} \mathrm{O} . \\
0,4539 \mathrm{~g} & , & 0,0572 & \mathrm{H}_{2} \mathrm{O} .
\end{array}
$$

Ber. für $\mathrm{C}_{10} \mathrm{H}_{18} \mathrm{~N}_{2} \mathrm{O}_{5}, 2 \mathrm{H}_{2} \mathrm{O}(282,19) \quad$ Gef.

$$
\begin{array}{llll}
\mathrm{H}_{2} \mathrm{O} & 12,77 & 12,75 & 12,61
\end{array}
$$

Zur Analyse und optischen Bestimmung war die im Vakuumexsiccator getrocknete Substanz nochmals über Phosphorpentoxyd unter $15 \mathrm{~mm}$ Druck bei $105^{\circ}$ getrocknet

$$
\begin{aligned}
& 0,3518 \mathrm{~g} \text { gaben } 0,6299 \mathrm{CO}_{\mathbf{2}} \text { und } 0,2268 \mathrm{H}_{2} \mathrm{O} . \\
& 0,2451 \mathrm{~g} \Rightarrow \quad 20,2 \mathrm{~cm} \mathrm{n} / 10-\mathrm{NH}_{\mathbf{s}} .
\end{aligned}
$$


198 Fischer und Fiedler, Derivate der Asparaginsäure.

$\begin{array}{lcr}\text { Ber. für } \mathrm{C}_{10} \mathrm{H}_{18} \mathrm{O}_{5} \mathrm{~N}_{2}(246,16) & \text { Gef. } \\ \mathrm{C} & 48,75 & 48,83 \\ \mathrm{H} & 7,37 & \mathbf{7 , 2 2} \\ \mathrm{N} & 11,38 & 11,54\end{array}$

Die wasserfreie Substanz schmilzt gegen $179^{\circ}$ (korr.182) unter Gasentwickelung. Die trockne Substanz ist hygroskopisch.

0,3952 g Substanz. Gesamtgewicht der wäBrigen Lösung 6,3577 g. $d^{13}=1,017$. Drehung im 1-dem-Rohr bei $18^{\circ}$ und Natriumlicht $1,71^{\circ}( \pm 0,02)$ nach rechts. Mithin

$$
[\alpha]_{\mathbb{D}}^{18}=+27,05^{0}( \pm 0,4) \text {. }
$$

0,3016 g Substanz. Gesamtgewicht 5,7722 g. $\quad d^{18}=1,015$. Drehung im 1-dcm-Rohr bei $18^{\circ}$ und Natriumlicht $1,42^{\circ}$ $( \pm 0,02)$ nach rechts. Mithin

$$
[\alpha]_{D}^{18}=+26,80^{\circ}( \pm 0,4) \text {. }
$$

Das früher als Leucylasparaginsäure ${ }^{1}$ ) beschriebene Präparat war entsprechend der Darstellung aus inaktiver Bromisocapronsäure noch ein Gemisch von Stereoisomeren. Infolgedessen weichen seine Eigenschaften etwas von den obigen Beobachtungen ab. Das gilt z. B. vom Krystallwasser, das früher nur $1 \mathrm{Mol}$. betrug, ferner vom optischen Verhalten, das früher nicht geprüft wurde. Herr Dr. Königs hat aber jetzt das Präparat nochmals nach dem alten Verfahren dargestellt und das Drehungsvermögen ganz anders, d. h. $[\alpha]_{D}=-4,6$, gefunden.

1) Ber. d. d. chem. Ges. 37, 4593 (1904). 